\title{
AUFHEBUNG VON STAATSVERTRÄGEN DURCH INNERSTAATLICHE GESETZE, DARGESTELLT AM FALLE INDONESIENS
}

\author{
Von Dr. Hellmuth Hecker
}

Ein zweites Mal hat Indonesien vor kurzem die ungewöhnliche Form gewählt, ein völkerrechtliches Vertragswerk durch innerstaatliches Gesetz außer Kraft zu setzen. Das erste Mal geschah dies, als Indonesien durch Gesetz Nr. 13 vom 21. 4./ 3. 5. $1956^{1}$ das Vertragswerk vom 2. 11.19492 über die holländisch-indonesische Union aufhob. In der Präambel des Gesetzes heißt es, Indonesien habe sich dazu entschlossen im Interesse von Staat und Volk, die durch die Verträge schwer benachteiligt worden seien. Indonesien habe die Niederlande öfter informiert, daß Inhalt und Zweck der Abkommen nicht länger gerechtfertigt seien, da West-Neuguinea nicht an Indonesien übertragen worden sei und da die Union mit den Niederlanden der Entwicklung Indonesiens im Wege stehe. Da zweiseitige Abmachungen keinen Erfolg erwarten ließen, sehe Indonesien keinen anderen Weg, als die Union in Úbereinstimmung mit seinen internationalen Rechten einseitig aufzuheben, und zwar rückwirkend ab 15. 2. 1956 (Art. 10). Von diesem Tage ab wurde das gesamte Vertragswerk für aufgehoben erklärt (Art. 1-5), insbesondere auch die Zusatzverträge zum Unions-Statut (Art. 4), die auch einen Vertrag über die Aufteilung der Staatsangehörigen enthielten ${ }^{3}$. Die Beziehungen zu den Niederlanden seien von nun an die zwischen voll souveränen Staaten auf Grund des Völkerrechts (Art. 6).

Dem Aufhebungsgesetz war ein Notenwechsel ${ }^{4}$ mit Holland vorausgegangen. Mit Note vom 14. 2. 1956 hatte Indonesien mitgeteilt, daß das Kabinett am Vortage die Auflösung der Union beschlossen habe und bat, die Hohen Kommissariate in Botschaften umzuwandeln. In einer weiteren Note vom 21. 2. 1956 datierte es die Auflösung auf den 15. 2. 1956. Die Niederlande antworteten darauf mit Note vom 3. 3. 1956: Durch die einseitigen Aufhebungen des 1949 unter Mithilfe der UNO feierlich geschlossenen Vertrages verstoße Indonesien gegen das Völkerrecht, denn die einseitige Aktion sei ungerechtfertigt, da die Niederlande Indonesien stets entgegengekommen seien und auch bereits durch Protokoll vom 10. 8. $1954^{5}$ die Union aufgehoben hätten. Die Niederlande müßten daher gegen Indonesiens einseitige Maßnahme protestieren.

Der zweite Fall dieser Art ereignete sich am 10. 4. 1969, als Indonesien den Doppelstaater-Vertrag mit der VR China vom 22. 4. 1955 aufhob ${ }^{6}$. Der Hintergrund hierfür war folgender: In Indonesien lebte seit langem eine große chinesische Minderheit. 1955 schloß Indonesien mit der Volksrepublik China einen ausführlichen Vertrag in 14 Artikeln 7 , der den betreffenden Chinesen ein Recht gab, durch Option die StA Chinas oder Indonesiens zu wählen. Durch Notenwechsel vom 3. 6. 1955 wurden in 5 Artikeln noch einige ergänzende Punkte geklärt ${ }^{8}$.

1 Englische Ubersetzung: State Papers Bd. 162, S. 981. Das Gesetz wurde am 3. 5. 1956 vom Präsidenten Indonesiens promulgiert.

2 UNTS Bd. 69, S. 3 ff.

3 Deutsche Übersetzung der 14 Artikel dieses Vertrages in: SGS Bd. 10, S. 74. Nach Ansicht von Sanger, Die Indonesische StA, Diss. Bonn 1959, S. 68 hat die StA-Regelung innerstaatlich in Indonesien Wirksamkeit behalten.

4 Englische Ưbersetzung der Noten 1 und 3 sowie französische Ubbersetzung der Note 2 in: State Papers Bd. 162, S. $983-985$.

5 Englischer Original-Text des Protokolls (10 Artikel) mit Notenwechsel: State Papers Bd. 161, S. 698-703. Nach Art. 4 war der StA-Vertrag in Kraft geblieben.

6 Deutsche Ưbersetzung des Gesetzes nebst amtlicher Begründung siehe unten.

7 Deutsche Ubbersetzung: Solich, Die Ưbersee-Chinesen in Südost-Asien Ffm 1960, S. 93 sowie Verträge der Volksrepublik China, Bd. XII/1, S. 101 der Schriftenreihe des Instituts für Asienkunde.

8 Englische UUbersetzung: State Papers Bd. 162, S. 421. 
Die Billigung des Vertrages verzögerte sich jedoch drei Jahre. Erst durch Gesetz Nr. 2 von $1958^{\circ}$ billigte Indonesien ihn und erließ dazu die sehr detaillierte DVO Nr. 20 vom 26. 5. 1959 in 24 Artikeln ${ }^{10}$. Wegen der damit immer noch nicht aus dem Weg geräumten Schwierigkeiten wurde am 16. 12. 1960 ein weiterer Vertrag ${ }^{11}$ zur Ausführung des ersten geschlossen (13 Artikel) und dazu wiederum eine indonesische DVO Nr. 5 von 1961 erlassen. Es wurden also drei internationale Abmachungen um drei nationale Rechtsvorschriften erlassen.

Dieses ganze mühsam erarbeitete Vertragswerk wurde durch das indonesische Gesetz von 1969 aufgehoben, jedoch nur ex nunc. Alle Optionen, die vorher auf Grund des Vertrages getätigt worden waren, wurden anerkannt und nur künftig wurde ein Optionsrecht beseitigt. Der Hauptgrund hierfür wird in Ziffer 4 der amtlichen Erläuterungen angegeben: Indonesien wollte nicht, daß Chinesen ein Recht erhielten, unter Aufgabe ihrer chinesischen StA nur die indonesische zu wählen, da es darüber kein Kontrollrecht besaß. Die Personen, die nicht optiert haben, bleiben also weiter Doppelstaater und Indonesien kann jederzeit die im Inland wohnenden Chinesen nur als Chinesen behandeln und ihnen die Anerkennung als Indonesier verweigern, da die Situation der Doppelstaatigkeit dieser Personen jetzt genauso ungeklärt ist wie vor dem Abkommen.

Sowohl die Aufhebung der Verträge mit Holland als auch die Aufhebung der Verträge der Volksrepublik China haben die Wirkung, daß sorgfältig ausgearbeitete Staatsangehörigkeitsabgrenzungen aus allgemeinen politischen Gründen aufgehoben werden, so daß eine erneute Unsicherheit auf dem Gebiete der Staatsangehörigkeit entsteht. Daß die Achtung vor dem Völkerrecht und insbesondere seinem obersten Grundsatz "pacta sunt servanda" durch solche einseitigen Akte gefördert würde, läßt sich schwerlich behaupten.

Gesetz Nr. 4 betreffend Unwirksamerklärung des Gesetzes Nr. 2 von 1958 (Staatsblad Nr. 5 von 1958) über den Doppelstaater-Vertrag zwischen der Republik Indonesien und der Volksrepublik China.

10. 4.1969

Amtl. Text: ind. Staatsblad 1969, Nr. 17

Engl. Úbersetzung: Warta Nr. 88 vom 18. 4. 1969, S. 845

Mit dem Segen des allmächtigen Gottes, hat der Präsident der Republik Indonesien, in der Erwägung:

daß auf Grund nationaler Interessen es als notwendig betrachtet wird, das

Gesetz Nr. 2 von 1958 betreffend Doppelstaater-Vertrag zwischen der Repu-

blik Indonesien und der VR China für nicht wirksam zu erklären, unter Berücksichtigung:

1. von Art. 5 (1), 20 (1), 26, 27 (1) der Verfassung von 1945;

2. von Gesetz Nr. 62 von 1958 (Staatsblad 1958 Nr. 113; Ergänzendes Staatsblad Nr. 1647)

folgendes Gesetz beschlossen:

Art. 1

Das Gesetz Nr. 2 von 1958 über den Doppelstaater-Vertrag zwischen der Republik Indonesien und der Volksrepublik China und seine Durchführungsvorschriften werden mit Wirkung vom Inkrafttreten dieses Gesetzes für unwirksam erklärt.

9 Indonesisches StBl Nr. 5 von 1958.

10 Indonesisches StBl Nr. 32 vom 1. 6. 1959, amtliche Begründung Suppl. Nr. 1765

11 Englischer Text: Circular des indonesischen Außenministeriums Nr. H 329 vom 23. 12.1960. 
Art. 2

Personen, die bei Inkrafttreten dieses Gesetzes die StA der Republik Indonesien auf Grund des Gesetzes Nr. 2 von 1958 über den Doppelstaater-Vertrag zwischen der Republik Indonesien und der VR China besaßen, bleiben Staatsangehörige der Republik Indonesien.

Art. 3

Diejenigen in Art. 2 genannten Personen, die bei Inkrafttreten dieses Gesetzes noch nicht volljährig waren, behalten ihre indonesische StA auch nach Erreichung der Volljährigkeit.

Art. 4

Die Vorschriften des Staatsangehörigkeitsgesetzes der Republik Indonesien, Gesetz Nr. 62 von 1958, werden auf die in Art. 2 und 3 erwähnten Personen angewandt.

Art. 5

Die in diesem Gesetz nicht ausreichend geregelten Angelegenheiten können durch den Justizminister näher geregelt werden.

Art. 6

Dieses Gesetz tritt mit dem Tage seiner Veröffentlichung in Kraft. Allen zu Kund und Wissen ist dieses Gesetz im Staatsblad der Republik Indonesien zu veröffentlichen.

\section{Amtliche Erläuterung}

Amtl. Text: Ergänzen des Staatsblad Nr. 2891 vom April 1969

Engl. Úbersetzung: Warta a. a. O. S. $846-848$

A. Allgemeines:

1. Der Doppelstaater-Vertrag zwischen der Republik Indonesien und der VR China vom 22. 4. 1955 ist durch Gesetz Nr. 2 von 1958 gebilligt worden. Am 15. 12. 1960 wurde ein Abkommen zum Verfahren der Durchführung geschlossen.

2. Die Durchführung des Vertrages wurde ausgeführt durch Regierungs-VO'en Nr. 20 von 1959 und Nr. 5 von 1961.

3. Obwohl es der Zweck besagten Vertrages war, das Problem der doppelten StA zwischen der Republik Indonesien und der VR China abschließend zu regeln, enthält er auch Vorschriften mit einer Sonderbehandlung für eine gewisse Gruppe, auf die das Gesetz Nr. 2 von 1958 für ziemlich lange Zeit gelten sollte.

Solche Vorschriften sind unter anderem:

a) Art. VI des Vertrages besagt, daß wer auch immer sowohl die StA der Republik Indonesien als auch der VR China besitzt, und bei Inkrafttreten des Vertrages noch nicht volljährig ist, innerhalb eines Jahres nach Volljährigkeit eine der beiden Staatsangehörigkeiten zu wählen hat.

Die Verwirklichung dessen bedeutet, daß es bis 1978 Personen geben wird, die die StA der VR China mit der der Republik Indonesien vertauschen können und umgekehrt.

Diese Angelegenheit beansprucht Zeit, Aufmerksamkeit und Verwaltung und ermöglicht Mißbräuche zu Lasten der Republik Indonesien.

b) Art 1(2) der Verfahrensvorschriften zur Durchführung des Abkommens besagt, daß Personen, die zwischen dem 27. 12. 1949 und 27. 12. 1951 noch nicht die Volljährigkeit erreichten und ihren Eltern folgend oder durch ihre Eltern 
die StA der Republik Indonesien aufgeben, als Personen mit doppelter StA betrachtet werden und berechtigt sind, eine der beiden Staatsangehörigkeiten zu wählen.

Sonderbehandlung auf Grund außerordentlicher Rechte und Möglichkeiten, die der übrigen Gruppe in der Gemeinschaft nicht zustehen, verstößt gegen das Prinzip der Gleichheit vor dem Gesetz, das von der Verfassung garantiert wird.

4. Prinzipiell erlaubt diese Vorschrift Ausländern, StA'e der Republik Indonesien zu werden, ohne daß die Regierung der Republik Indonesien ein Prüfungsrecht erhält, wodurch daher die Sicherheit der Republik Indonesien gefährdet werden kann.

5. Durch die Unwirksamkeitserklärung des Gesetzes Nr. 2 von 1958 und all seiner Durchführungs-Vorschriften können diese Vorschriften nicht länger angewandt werden.

6. Personen, die auf Grund der Vorschriften des Vertrages eine StA gewählt haben, bleiben im Besitz der gewählten StA.

Kinder, die bei Inkrafttreten dieses Gesetzes noch nicht volljährig sind, können bei Erreichung der Volljährigkeit keine andere StA mehr wählen und sie folgen der StA ihrer Eltern. Dies schließt nicht aus, daß die Betreffenden ihre StA auf Grund der geltenden Gesetzesvorschriften ändern.

7. Die Verzögerung diplomatischer und konsularischer Beziehungen zwischen der Republik Indonesien und der VR China beeinflußt, ja verhindert die Ausführung besagten Doppelstaater-Vertrages.

\section{B. Artikelweise:}

\section{Art. 1: Selbstverständlich}

Art. 2: Außer Personen, die die StA der Republik Indonesien durch Abgabe einer Erklärung vor Gericht oder einer indonesischen Auslandsvertretung gewählt haben, sind in diesem Artikel eingeschlossen diejenigen, die Formblätter C und D auf Grund der Regierungs-VO'en Nr. 20 von 1959 und Nr. 5 von 1961 erhielten.

Art. 3: Siehe Allgemeine Erläuterungen Nr. 6 Satz 2.

Art. 4: $\mathrm{Da}$ alle in Art. 2 und 3 erwähnten Personen StA'e der Republik Indonesien sind, sind auf sie die Vorschriften des StA-Gesetzes Nr. 62 von 1958 und über den Verlust der StA der Republik Indonesien voll anzuwenden.

Art. 5 und 6: Selbstverständlich. 